\title{
An islet of social ability in Asperger Syndrome: Judging social attributes from faces
}

\author{
Sarah White ${ }^{\text {a }}$, Elisabeth Hill ${ }^{\text {a,b }}$, Joel Winston ${ }^{\mathrm{c}}$, Uta Frith ${ }^{\mathrm{a}, *}$ \\ ${ }^{a}$ Institute of Cognitive Neuroscience, University College London, UK \\ ${ }^{\mathrm{b}}$ Goldsmiths College, University of London, UK \\ ${ }^{\mathrm{c}}$ Wellcome Department of Imaging Neuroscience, University College London, UK
}

Accepted 9 December 2005

Available online 3 February 2006

\begin{abstract}
We asked adults with Asperger Syndrome to judge pictorial stimuli in terms of certain social stereotypes to evaluate to what extent they have access to this type of social knowledge. Sixteen adults with Asperger Syndrome and 24 controls, matched for age and intelligence, were presented with sets of faces, bodies and objects, which had to be rated on a 7-point scale in terms of trustworthiness, attractiveness, social status, and age, or, in the case of objects, price. Despite impaired performance on two important aspects of social cognition (second-order mentalizing and face recognition) the social judgements of the individuals with Asperger Syndrome were just as competent and consistent as those of their matched controls, with only one exception: there was a trend for them to be less able to judge the attractiveness of faces if they were the same sex. We explain this difference in terms of a weakness in mentalizing, specifically the ability to take a different point of view: While all other stereotypic attributions could be made from an egocentric point of view, judging the attractiveness of faces of one's own sex requires taking the perspective of someone of the opposite sex, a challenge for people with mentalizing problems. We conclude that individuals with Asperger Syndrome show preserved aspects of social knowledge, as revealed in the attribution of stereotypes to pictures of people. These findings suggest that there are dissociable subcomponents to social cognition and that not all of these are compromised in Asperger Syndrome.
\end{abstract}

(C) 2006 Elsevier Inc. All rights reserved.

Keywords: Asperger Syndrome; Social cognition; Mentalizing; Theory of mind; Face recognition; Stereotypes; Attractiveness; Trustworthiness; Status

\section{Introduction}

The social impairments present in autism-spectrum disorders form part of the essential diagnostic criteria used to define such conditions (DSM-IV; American Psychiatric Association, 2000). Asperger Syndrome (AS) is widely acknowledged to be a variant of autistic disorder, without a delay in language and cognitive development. Children with AS are typically diagnosed in later childhood (Macintosh \& Dissanayake, 2004). The later diagnosis may be partially explained by the presence of good language and often outstanding intellectual abilities, which can mask deficien-

\footnotetext{
* Corresponding author. Fax: +4402078132835.

E-mail address: u.frith@ucl.ac.uk (U. Frith).
}

cies in social development and cognitive flexibility (Frith, 2004).

Among the social impairments documented in AS are mentalizing problems (e.g., Baron-Cohen, Jolliffe, Mortimore, \& Robertson, 1997; Baron-Cohen, O'Riordan, Stone, Jones, \& Plaisted, 1999; Castelli, Frith, Happé, \& Frith, 2002; Happé, 1994; Heavey, Phillips, Baron-Cohen, \& Rutter, 2000; Kleinman, Marciano, \& Ault, 2001; Rutherford, Baron-Cohen, \& Wheelwright, 2002). However, these impairments typically only involve difficulties with second-order-, but not first-order-, mental state attribution (Baron-Cohen, 1989; Ziatas, Durkin, \& Pratt, 1998).

Face processing is one area of social cognition that has recently received much attention by autism researchers (see Grelotti, Gauthier, \& Schultz, 2002, for a review). Faces hold a wealth of information about the identity 
of the person and their group membership. Even if of neutral expression, they give vital cues to the initiation or avoidance of social contact, and appropriate mode of approach. A variety of impairments in face processing have been revealed specifically in people with high-functioning autism or AS.

Some of these impairments involve mentalizing. For example, individuals with AS or high-functioning autism are poor at predicting the intentions or mental states of others from pictures of their eyes (Baron-Cohen et al., 1997; Baron-Cohen, Wheelwright, Hill, Raste, \& Plumb, 2001). However, there are also impairments that are likely to be of separate origin. Thus, there are problems in face recognition memory (Blair, Frith, Smith, Abell, \& Cipolotti, 2002) and emotional expression understanding (Hobson, 1993). Additionally, the scan path of eye gaze when looking at faces can give clues as to the origin of these problems. Individuals with autistic disorder have been shown to have a preference for attending to the mouth region of the face when the bias in normal development is for the eyes (Joseph \& Tanaka, 2003; Klin, Jones, Schultz, Volkmar, \& Cohen, 2002). If they avoid looking at the eyes, they would therefore miss important communicative signals conveyed by the eye regions, such as emotional expression.

Emotional responsiveness and emotion regulation are also areas where impairments have been noted (Hill, Berthoz, \& Frith, 2004; Shamay-Tsoory, Tomer, Yaniv, \& Aharon-Peretz, 2002). However, many aspects of social cognition, such as implicit imitation of action, empathy for others' feelings, and the use of speech and gesture in two-way communication, still remain to be investigated in people with AS. It seems possible that in view of their varied clinical picture, which can include milder forms with more subtle impairments, some aspects of social knowledge may show preserved functioning. Certainly, there is ample biographical evidence that adults with AS can be highly skilled at learning and applying social rules (e.g., Gerland, 1997; Lawson, 1998; Sainsbury, 2000), and in autistic disorders in general there is evidence of successful teaching of social skills (e.g., Silver \& Oakes, 2001).

One aspect of social cognition, the ability to use social stereotypes, has as yet hardly been studied in autism-spectrum disorders, although it has been a major focus of research in traditional social psychology. Stereotypes allow us to categorise people we have never met before, thus simplifying and organising social information. For example, we are prepared to give a preliminary judgement as to whether a person is attractive, trustworthy, or has high social status from minimal visual cues. Knowledge of stereotypes is thought to be culturally transmitted and typically emerges from about the age of 3 years (Hirschfeld, 1996). Importantly, stereotyping allows us to prepare for potential social interaction by making predictions about a person's behaviour on the basis of their group membership (Mackie, Hamilton, Susskind, \& Rosselli, 1996). Stereotypes therefore may help us to decide from a photograph whether we may wish to avoid or to meet an unfamiliar person independently of what that person is like as an individual.

One of the first neuropsychological studies to exploit the ability to make quick judgments of people on the basis of photographs was carried out by Adolphs, Tranel, and Damasio (1998). They showed a set of 100 faces to three patients with bilateral amygdala damage and asked them to rate on a 7-point scale, either approachability (how much they wanted to walk up to a person and strike up a conversation with them) or, in another session, trustworthiness (how much they would trust that person with all their money or with their life). Since the attribution of these characteristics to photographs is subjective, the authors established a reference sample of 46 undergraduates to rate this set of faces and used these ratings as a standard against which to compare the ratings of the patients. They found that the patients rated the 50 most negative faces more positively compared to ratings given by the control sample, and also by 10 patients with other types of brain damage. This was true for both trustworthiness and approachability, whereas they gave similarly positive ratings to the faces that the control sample judged as the 50 most positive.

Adolphs, Sears, and Piven (2001) used the same task with individuals with AS and found that these individuals performed similarly to the amygdala patients, also judging the 50 most untrustworthy faces as more trustworthy than controls while rating the most trustworthy ones equally positively. However, somewhat surprisingly, they performed as well as controls in their judgment of approachability. Adolphs and colleagues concluded that individuals with AS, like patients with bilateral amygdala lesions, show less ability to discriminate faces in terms of subjectively perceived trustworthiness with a bias towards strong positive ratings in some individuals.

Just what perceptual information we use from the photographs to make these judgements is not clear, but the attribution of social traits such as trustworthiness and approachability is likely to draw on social knowledge, specifically, on culturally acquired knowledge about stereotypes. There are a number of reasons to think that this is likely to be an area of difficulty in AS. First, if some basic perceptual aspects of face processing are impaired or at least abnormal in individuals with AS, this may prove a disadvantage in acquiring such knowledge. Second, if a degree of mentalizing impairment is present, then individuals with AS would be expected to be less able to acquire knowledge of stereotypes, given that this knowledge is socially transmitted.

Bearing in mind the mixed results from the Adolphs et al. (2001) study, we explored further dimensions of important social stereotypes using their paradigm of judging faces. In particular, we wished to explore the dimensions of attractiveness and social status, but we also included trustworthiness to replicate the findings from their original study. 


\section{Method}

\subsection{Participants}

In total, 24 control adults (12m:12f) and 16 adults with AS (10m:6f) took part in the study, aged between 18 and 63 years and with intelligence in the normal range (FSIQ $\geqslant 85$ ). The groups were matched for gender $(\chi=0.606)$ and age $(t(38)=1.280)$ as well as verbal, performance and full-scale IQ $(t(38)<1)$ (see Table 1). Participants were recruited through adverts placed in local community centres, as well as in Asperger Support Group newsletters and on web pages. All participants in the experimental group had previously received a diagnosis of AS from a clinical psychologist or a psychiatrist. To support their diagnoses we used the Autism-Spectrum Quotient (AQ) questionnaire (Baron-Cohen, Wheelwright, Skinner, Martin, \& Clubley, 2001). The adults with AS showed a significantly elevated score on the AQ $(t(37)=7.98$, $p<.001$ ), and all but three of the adults with AS were above the suggested cut-off of 32 on this measure, while none of the controls were.

The study obtained ethical approval from the Joint UCL/UCLH committees on the Ethics of Human Research and informed consent to participate was obtained from each adult.

\subsection{Procedure}

All participants were tested individually in a quiet room at the Institute of Cognitive Neuroscience. Testing was divided into two sessions of about $1 \frac{1}{2} \mathrm{~h}$ each and all participants completed a battery of standard tasks, to provide background information on both their general ability and social functioning, as well as the experimental tasks.

Verbal and performance IQ were assessed by means of the Wechsler Adult Intelligence Scale (WAIS-III ${ }^{\mathrm{UK}}$; Wechsler, 1999). Standard first- and second-order false belief tasks ('Sally-Ann,' Baron-Cohen, Leslie, \& Frith, 1985; 'Coat Story,' Bowler, 1992) and an advanced test of mentalizing (Strange Stories-revised version; Happé et al., 1996) were administered.

As some of the experimental tasks involved the observation of faces, the Benton Facial Recognition Task (Benton, Sivan, Hamsher, Varney, \& Spreen, 1994) was included to look for any visuo-perceptual deficits in the processing of

Table 1

Means (and standard deviations) for group demographics

\begin{tabular}{lcc}
\hline & Control & AS \\
\hline$N$ (male:female) & $24(12: 12)$ & $16(10: 6)$ \\
Age (years) & $37.75(12.44)$ & $32.31(14.20)$ \\
Verbal IQ & $117.13(12.65)$ & $113.13(16.37)$ \\
Performance IQ & $110.92(12.87)$ & $108.00(13.71)$ \\
Full-scale IQ & $115.25(11.35)$ & $111.81(15.60)$ \\
AQ $(p<.001)$ & $16.65(6.81)$ & $34.63(7.08)$ \\
\hline
\end{tabular}

Table 2

Summary of stimulus sets and attributes judged from them

\begin{tabular}{llll}
\hline & Type of stimulus & Attributes judged & Source \\
\hline Set 1 & Faces & Trustworthiness, age & $\begin{array}{l}\text { Winston et al. } \\
(2002)\end{array}$ \\
Set 2 & Faces & Attractiveness, age & $\begin{array}{l}\text { O'Doherty et al. } \\
(2003)\end{array}$ \\
Set 3 & Faces & Socioeconomic status, age & Novel \\
Set 4 & $\begin{array}{l}\text { People with } \\
\text { blurred faces }\end{array}$ & Socioeconomic status, age & Novel \\
Set 5 & Objects & Price & Novel \\
\hline
\end{tabular}

faces that might otherwise explain any group differences on the experimental tasks.

\subsubsection{Experimental tasks}

Nine tasks, corresponding to nine experimental conditions, were included. Each task consisted of a set of 48 stimuli, presented one by one on a laptop computer using E-prime software. All participants responded to all stimuli in all conditions. Each condition was presented as a separate block, and both the blocks and the stimuli within each block were presented in a random order. The participants were asked to judge each picture for a particular attribute on a scale ranging from 1 (low) to 7 (high), with 4 as the mid point; this scale was chosen to increase task difficulty and avoid ceiling effects that were likely from a binary response (yes/no). Five different stimulus sets were used and these were either photos of faces, of people with their faces obscured, or of objects. The attributes to be judged from the faces and people were trustworthiness, attractiveness, socioeconomic status and age, whilst price was judged for each object (see Table 2). All stimuli were greyscale images, the faces and people were frontal images and the faces were all Caucasian ${ }^{1}$ with neutral expressions and without clothes or hats. Each picture was presented for a maximum of $10 \mathrm{~s}$, although participants were encouraged to respond as quickly as possible.

To facilitate the investigation of face processing, Perrett and his colleagues (e.g., Perrett, May, \& Yoshikawa, 1994) have created a large data base of face stimuli, which has been widely used in research. We adopted some of these stimuli, which had been previously rated for the relevant social attributes to form two of the sets of images we used. The judgment of attractiveness was elicited from a set of faces, for which O'Doherty et al. (2003) had collected ratings from 23 university students and staff, with an average age of 24 years. This was the only set to contain both male and female individuals (an equal number of each), as opposed to all male individuals in all other sets of images.

\footnotetext{
${ }^{1}$ The participants in both groups were also overwhelmingly Caucasian; one adult with AS was half Oriental and one control was Asian.
} 
For the judgement of trustworthiness we used a subset of those for which Winston, Strange, O'Doherty, and Dolan (2002) had collected ratings from 30 adults, also university students and staff, also with an average age of 24 years.

We devised a novel set of faces for the judgement of socioeconomic status. To control for the possibility that poor face processing might impact on social judgements from faces, we also constructed a set of greyscale photographs of whole persons with blurred faces. Here, social status had to be inferred from clothes and/or body posture rather than facial characteristics. Because ratings of social status from these stimuli is entirely subjective, we asked 14 university students and staff, from the same pool as those who had rated the sets of faces for attractiveness and trustworthiness in previous studies, to rate the faces and bodies on the attribute of social status. None of these raters were used in the control sample in our experiment, and so these ratings constitute an independent standard for the various social attributes that are subjectively attributed to the stimuli used. We will refer to them as reference samples.

Age judgements from all four sets of photos were also included. These are more likely to be based on physical features. Here absolute values were ascribed to each of the seven points of the scale $(1=$ less than 10 years; $2=10$ 19 years; $3=20-29$ years; $4=30-39$ years; $5=40-49$ years; $6=50-59$ years; $7=60$ years or more). Because the judgment of age is still subjective, we used again reference samples of between 6 and 13 individuals whom we

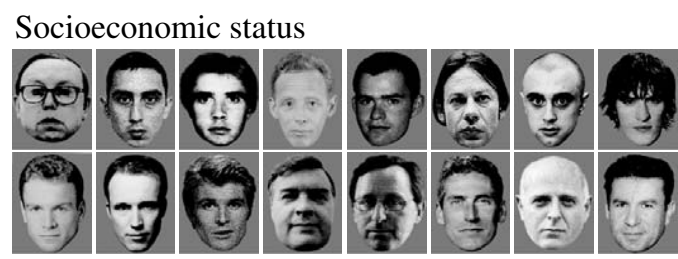

Socioeconomic status
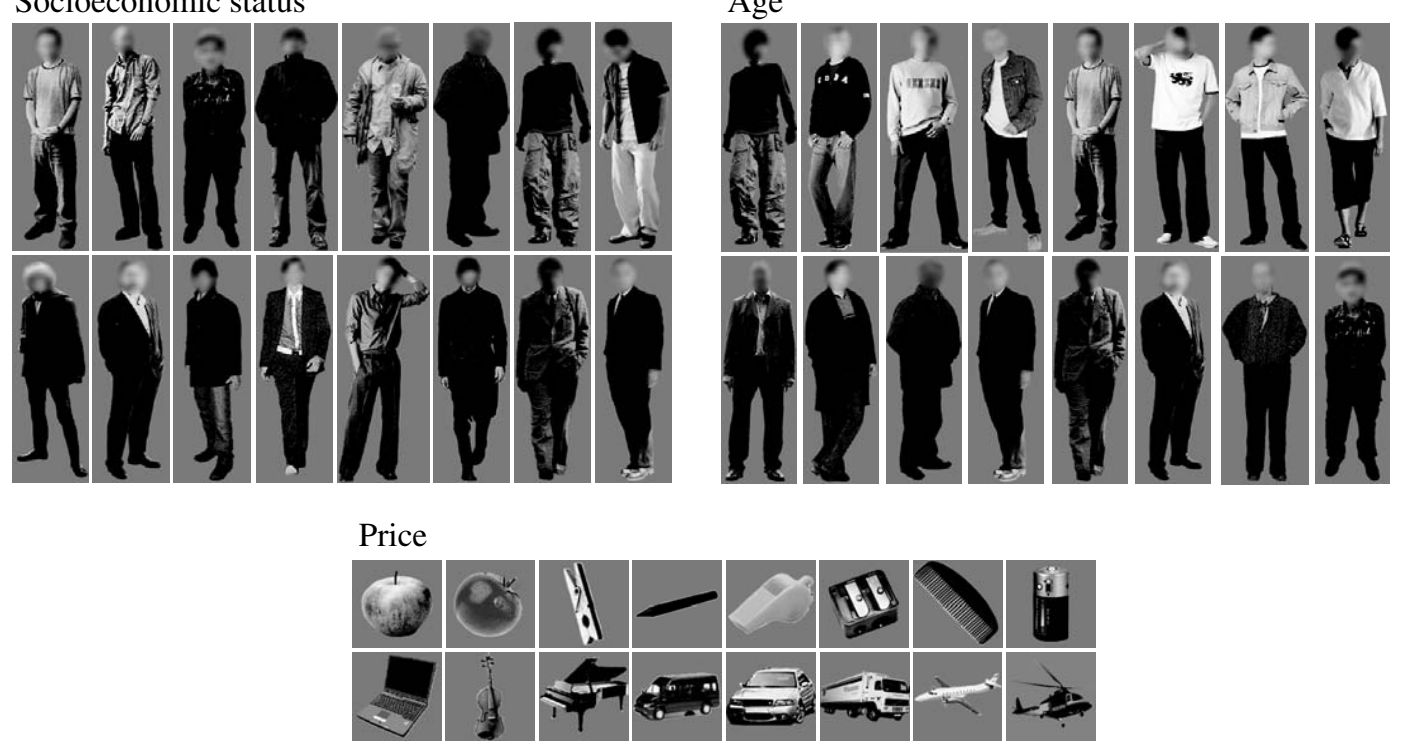

Fig. 1. End point stimuli; the 8 lowest (top line) and 8 highest (bottom line) ranked stimuli from the reference group for socioeconomic status and for price of objects. 
differences would be expected between the ranks given to the extremes. As the attractiveness set contained female pictures as well as male pictures, we selected the four most extreme males and the four most extreme females.

As already mentioned, all participants ranked all 48 stimuli in each condition. We extracted the rankings they gave to the eight lowest and eight highest ranked stimuli as defined by the reference samples. We then calculated the difference in mean ranking between these endpoint stimuli for each participant and used paired samples $t$ tests to compare the two groups' performance for each of the nine conditions.

We did not compare the absolute values of the difference between endpoint stimuli across attributes, as no attempt had been made to equate the range of the attributes in the different sets. Thus, certain attributes would be expected to produce smaller differences due to a lower range of the attribute in that particular set. This does not reflect a lesser ability to discriminate. For example, age had a rather small range of differences in the set of faces rated for attractiveness. Nevertheless the faces judged youngest and oldest by the reference samples were also differentiated by our participants, with a mean difference of around 1 point on the given 7-point scale. While a difference score of 0 indicates no differentiation between high and low stimuli, a difference score above 0 indicates at least some degree of ability to differentiate. Possible scores range from -6 to +6 , although such extreme scores are unlikely, and negative scores indicate a reverse preference compared to the reference sample.

We wish to emphasize that we cannot use this score to deduce the absolute level of ability to make the various attributions. To do this it would be necessary to have normative data and a number of different stimulus sets to assess whether performance was independent of specific stimulus characteristics. Thus, we are not making any claims as to individual differences in competence. Instead our data address the question of differences in group abilities to differentiate between independently ranked lowest and highest pictures in a given set on a given dimension. Thus, if individuals with AS were to obtain a significantly smaller score compared to the controls, we would infer that their ability to use a given social attribute is impaired.

\section{Results}

Compared to the age and IQ matched control group, the adults with AS showed impairments in mentalizing as a group, a typical result. They were impaired at the secondorder $(\chi=6.667, p=.010)$, but not first-order $(\chi=$ 1.538), false belief task as well as at the advanced test of mentalizing $(t(38)=2.042, p=.048)$, although there was a high degree of overlap in individual results with the control group (AS range: 5-16; control range: 10-16). The adults with AS were also impaired as a group relative to controls on the Benton Facial Recognition Task $(t(18.57)=3.599, p=.002)$, indicating poor perceptual processing of faces (see Table 3 ), although there was again
Table 3

Means (and standard deviations; unless otherwise stated) of group results from background tests

\begin{tabular}{lll}
\hline & Control & AS \\
\hline First-order FB $(\% \text { passing })^{*}$ & 100 & 93.75 \\
Second-order FB (\% passing) $^{*}$ & 100 & 75 \\
Advanced theory of mind test $^{*}(\max =16)$ & $13.79(1.69)$ & $12.31(2.89)$ \\
Benton $^{* *}$ & $49.71(3.16)$ & $44.14(5.26)$ \\
\hline${ }^{*} p<.05$. & & \\
${ }^{* *} p<.01$. & & \\
$p<$
\end{tabular}

Table 4

Means (and standard deviations) of the difference between endpoint stimuli for each group on each condition

\begin{tabular}{lll}
\hline & Control & AS \\
\hline Faces Set 1: Trustworthiness & $1.35(0.55)$ & $1.06(0.99)$ \\
Faces Set 1: Age & $2.14(0.61)$ & $2.26(0.62)$ \\
Faces Set 2: Attractiveness & $2.76^{*}(0.95)$ & $1.98^{*}(1.11)$ \\
Faces Set 2: Age & $1.01(0.42)$ & $0.94(0.36)$ \\
Faces Set 3: Status & $1.55(0.69)$ & $1.45(0.96)$ \\
Faces Set 3: Age & $2.15(0.56)$ & $2.25(0.41)$ \\
People: Status & $1.72(0.74)$ & $1.72(1.09)$ \\
People: Age & $2.47(0.79)$ & $2.43(0.99)$ \\
Objects: Price & $5.25(0.28)$ & $5.12(0.42)$ \\
Attractiveness: same sex & $2.75^{* *}(0.93)$ & $1.64^{* *}(1.43)$ \\
Attractiveness: opposite sex & $2.77(1.19)$ & $2.31(1.07)$ \\
\hline
\end{tabular}

${ }_{* *}^{*} p<.05$.

${ }^{* *} p<.01$

overlap in performance between the groups (AS range: 36-50; control range: 42-54).

As can be seen in Table 4, the group with AS and the controls performed remarkably similarly when rating the different sets of images in terms of social attributes. Only one group difference was found in the ratings of the nine picture sets. This occurred when judging attractiveness from faces. The individuals with AS had a smaller difference in their ranks given to the endpoint stimuli compared to the controls $(t(38)=2.390, p=.022)$, indicating that they were differentiating less well between low and high stimuli. However, we note that if a correction for multiple comparisons was applied to the four critical conditions (leaving out the control conditions of age and price), this difference would no longer be significant $(p=.088)$. There was also a high degree of overlap in the range of scores (AS range: -0.25 to 3.63 ; control range: $1.5-4.63$ ). No other differences were found in any other condition (see Table 4 and Fig. 2).

Correlations within the group of adults with AS were calculated between the difference scores for the four critical conditions and were found to be significant between trustworthiness, status from faces and status from people $(p<.05)$. Attractiveness, however, was not correlated to any of these measures. Correlations between performance on these social attribution tasks, the higher-order mentalizing task, the face perception task and IQ were also calculated in the group of adults with AS. Verbal IQ was unsurprisingly correlated to performance on the higher-order 


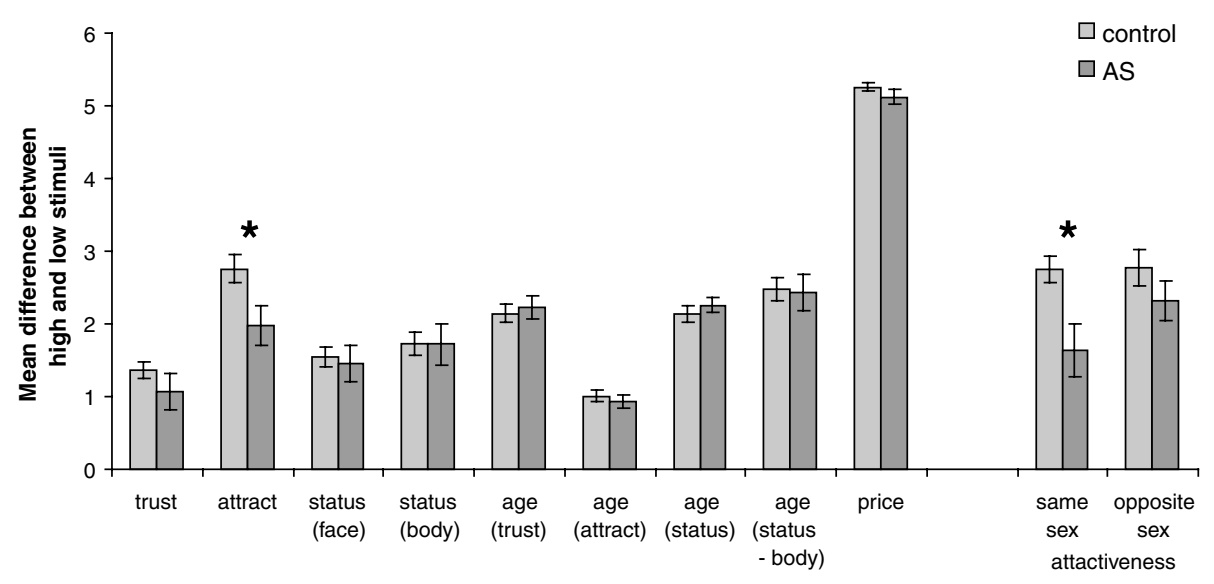

Fig. 2. Graph showing the difference between endpoint stimuli for each group on each condition; error bars show standard error of the mean. ${ }^{*} p<.05$.

mentalizing task $(p=.013)$ but no other correlations were significant. However, it should be noted that these correlations would no longer be significant if a correction for multiple comparisons was applied.

To investigate whether the individuals with AS were defining attractiveness differently to controls and therefore appeared to be worse at judging attractiveness, intra-group agreement was calculated for each group using Kendall's coefficient of concordance. Although both groups showed high intra-group agreement (AS, $W(47)=0.416, p<.001$; control, $W(47)=0.654, p<.001)$, the level of agreement was lower within the group of individuals with AS (value of $W$ closer to 0 ). This difference in levels of agreement was found to be significant ( $p=.007$ ), using a multiple permutations test. $^{2}$

One difference between the attractiveness stimulus set and the other face sets was that it was the only set to contain both male and female faces. The attractiveness data was therefore reanalysed, after separating judgements into those when judging a face of the same sex or of the opposite sex. This comparison revealed that the individuals with AS were only worse than the controls when judging faces of the same sex, discriminating between high and low attractiveness faces less well than controls $(t(38)=2.978$, $p=.005$ ) (see Table 4 and Fig. 2). Further analysis revealed that this difference arose from the individuals with AS finding the highly attractive same sex faces less attractive than controls did $(t(38)=2.676, p=.011)$. Furthermore, the controls were as good at judging the attractiveness of faces of the same or opposite $\operatorname{sex}(t(23)=0.104)$ whereas the AS adults were worse at judging faces of the same sex than the opposite $\operatorname{sex}(t(15)=2.234, p=.041)$.

As a more complete replication of the Adolphs et al. (2001) results, the data from the trustworthiness judgements

\footnotetext{
${ }^{2}$ This method involves the random allocation of participants to the two groups 1000 times, recalculating each time the difference between the Kendall's $W$ for the two groups, and thus creating a null distribution. The difference between Kendall's $W$ for the two actual groups is then compared to the null distribution and the probability of this difference occurring is calculated.
}

were reanalysed using a control group similar to that used in their study. Their control group consisted of 46 university undergraduates (see Adolphs et al., 1998), a population similar to that used in Winston et al. (2002), whilst our control group was carefully matched to our group of adults with AS on measures of intelligence and age. In addition, Adolphs et al.'s stimuli were ranked on the basis of their control group's trustworthiness ratings rather than on the basis of an independent reference sample's ratings. This created the greatest possible difference between high and low stimulus means for their control group, thus biasing any other group to produce a smaller difference, and therefore increasing the likelihood of finding a difference between the groups. Conversely, the present study used a separate group of controls external to the experiment (the reference sample in Winston et al., 2002) to rank the stimuli, thus minimising the chance of producing a false positive result.

The trustworthiness data were therefore reanalysed using the Winston et al. (2002) reference sample $(n=30)$ for both the ranking and as the comparison control group. Using this approach, our data reveal the same results as Adolphs et al. (2001), with the adults with AS discriminating more poorly between the endpoint stimuli $(t(44)=3.688, p=.001)$, ranking the low faces as higher than the controls $(t(44)=5.349 . p<.001)$ but being comparable on the high faces $(t(44)=0.382)$. Furthermore, if the same comparison is made between the Winston et al. (2002) reference sample and the present control group, the same difference is found with the present controls being worse at discriminating end points $(t(45.8)=3.972, p<.001)$, judging the low faces as higher than the controls $(t(52)=5.789, p<.001)$ but not the high faces $(t(50.1)=0.571) .{ }^{3}$ We believe that this replica-

\footnotetext{
${ }^{3}$ One further small difference between the present study and that of Adolphs et al. (2001) was in the method of data analysis. Further analysis revealed that using their slightly different method produced identical results to ours with the trustworthiness stimuli (ranking the stimuli on the basis of the reference group instead of the controls); no difference was found between the groups for either the low $(t(38)=0.495)$ or high $(t(38)=1.175)$ stimuli. Any differences between our results and those of Adolphs et al. (2001) therefore cannot be assumed to be due to the method of analysis used.
} 
tion shows that our results are comparable to those of Adolphs et al. (2001), but we propose that our more stringent control procedure is more appropriate. If so, the previous claim of a group difference in the judgement of trustworthiness must be considered with caution.

\section{Discussion}

Despite poor facial perception as assessed by the Benton Facial Recognition Task, and poor mentalizing abilities, as evident in an advanced story comprehension test, the present group of individuals with AS were just as competent in making social judgements from photographs as a group of ordinary individuals matched for age and IQ. This result is surprising for two reasons. First, despite their impairments in face recognition, they did not differ from the controls when attributing a social stereotype, such as status, to faces or to whole bodies. Second, despite their impairments in mentalizing, they did not differ from the controls when attributing social stereotypes, such as status or trustworthiness, or a more physical construct such as age.

The correlational trends between performance on the different social attributes within the AS group suggest that these tasks may be tapping the same underlying ability. On the other hand, there were no such correlational trends between performance on these social attributes and our other two measures of social competence, face recognition and stories requiring theory of mind reasoning. This supports the idea that the judgement of stereotypic social attributes is independent from other aspects of social cognition.

Our findings imply that access to knowledge of social stereotypes was equally available to our group of individuals with AS as to our group of controls, and in particular that it is not necessary for two important aspects of social cognition, mentalizing and face perception, to be intact for the ability to judge social attributes from pictures of faces. How can the surprising competence of our socially impaired individuals with AS be explained? Hirschfeld (1995) suggested that knowledge of stereotypes is encompassed by a domain of naïve sociology that is separate to naïve psychology. In contrast to naïve psychology, which is assumed to rest on the ability to mentalize, naïve sociology is assumed to rest on the ability to reason about group membership. Thus, to acquire and use social stereotypes requires the capacity to understand others in terms of their group membership and the characteristics that are representative of a group. If this ability is based on a separate cognitive mechanism, as Hirschfeld suggests, then it is entirely possible that despite mentalizing and face perception impairments, individuals with AS have a preserved ability to attribute social stereotypes to photographs.

This possibility is in line with anecdotal evidence which suggests that individuals with AS may show implicit understanding of social stereotypes. For example, when they aspire to have a partner, they often specify that this must be an attractive partner, such as a movie star, while their family may try in vain to induce more realistic expectations. Furthermore, there is evidence from the scant previous literature on attributional processes in AS, suggesting that mentalizing abilities and attributional processes may be distinct (Blackshaw, Kinderman, Hare, \& Hatton, 2001), although it should be noted that this study uses a very different paradigm to the present one and has very different aims.

The only difference between our AS group and controls was found when judging attractiveness. In fact, this impairment was only present when judging the attractiveness of someone of the same sex: AS adults judged highly attractive faces of the same sex less attractive than controls. While they were still able to discriminate between the attractive and unattractive faces, they were less accurate than controls at such judgements, and less accurate than when they judged faces of the opposite sex.

What could be the cause of such a specific deficit? We suggest that it could in fact be due to mentalizing problems. Same sex attractiveness judgements are different from all the other judgements because they are the only ones that require a change in point of view, which is a challenge for people with mentalizing difficulties: You need to take the point of view of someone of the opposite sex to give an appropriate judgement of the attractiveness of someone who is the same sex as you. In contrast, the judgement of social status, trustworthiness and attractiveness of the opposite sex can be made from an egocentric perspective, i.e., simply based on one's own attitude to each picture without taking someone else's perspective. If making such judgements from an egocentric point of view, you might be inclined to simply give your own attitude toward the picture and thus judge highly attractive faces of the same sex as less attractive than those of the opposite sex in the same set of pictures. Given their problems in mentalizing, the individuals with AS may not have based their judgement on a change in perspective, and thus produced less consistent and less typical results. There is reason to believe that normal adults, when judging a face of the same sex, do so in relation to their own attractiveness (Buss, 1992). The present data hint that individuals with AS are inclined to judge highly attractive faces of the same sex as less attractive, implying perhaps that in their self perception they are highly attractive.

Overall, our results demonstrate that social knowledge is far from absent in individuals with AS. In particular, they suggest that culturally transmitted knowledge about stereotypes and group membership (Hirschfeld, 1996) can be acquired by individuals with AS who are socially impaired. However, the present study only informs us about the ability to use stereotypic knowledge from pictures; it does not tell us to what extent this knowledge is used in everyday life. This is a challenging question that now needs to be investigated. At the very least, this study suggests that even with impaired mentalizing, studying pictures of people and talking about them as members of a social group may be a 
useful aid to social learning, and this is indeed a feature of many social skills training programmes. This study supports the notion that social cognition has many different cognitive components and that there are aspects of social cognition that are spared in AS.

\section{Acknowledgments}

This study was funded by a Medical Research Council Grant G9617036 to U.F. The study was facilitated by an MRC Cooperative Grant at UCL-ICN. We are grateful to David Perrett for permitting the use of his stimulus sets, and John O'Doherty for allowing us to use his data on the set of faces ranked for attractiveness. We also thank Antonia Hamilton, Will Penny, and Chris Frith for their help with the analysis, and Gemma Norton, Zoe Fortune, and Philip Angell for their help with data collection.

\section{References}

Adolphs, R., Sears, L., \& Piven, J. (2001). Abnormal processing of social information from faces in autism. Journal of Cognitive Neuroscience, 13, 232-240.

Adolphs, R., Tranel, D., \& Damasio, A. (1998). The human amygdala in social judgment. Nature, 393, 470-474.

American Psychiatric Association (2000). Diagnostic and statistical manual of mental disorders (4th ed.), text revision. Washington, DC: American Psychiatric Association.

Baron-Cohen, S. (1989). The autistic child's theory of mind: A case of specific developmental delay. Journal of Child Psychology and Psychiatry, 30, 285-297.

Baron-Cohen, S., Jolliffe, T., Mortimore, C., \& Robertson, M. (1997). Another advanced test of theory of mind: Evidence from very high functioning adults with autism or Asperger syndrome. Journal of Child Psychology and Psychiatry, 38, 813-822.

Baron-Cohen, S., Leslie, A., \& Frith, U. (1985). Does the autistic child have a "theory of mind"? Cognition 21, 37-46.

Baron-Cohen, S., O'Riordan, M., Stone, V., Jones, R., \& Plaisted, K. (1999). Recognition of faux pas by normally developing children and children with Asperger syndrome or high-functioning autism. Journal of Autism and Developmental Disorders, 29, 407-418.

Baron-Cohen, S., Wheelwright, S., Hill, J., Raste, Y., \& Plumb, I. (2001). The "Reading the Mind in the Eyes" Test revised version: A study with normal adults, and adults with Asperger syndrome or high-functioning autism. Journal of Child Psychology and Psychiatry, 42, 241-251.

Baron-Cohen, S., Wheelwright, S., Skinner, R., Martin, J., \& Clubley, E. (2001). The autism-spectrum quotient (AQ): Evidence from Asperger syndrome/high-functioning autism, males and females, scientists and mathematicians. Journal of Autism and Developmental Disorders, 31, 5-17.

Benton, A., Sivan, A., Hamsher, K., Varney, N., \& Spreen, O. (1994). Benton Facial Recognition Test. Florida: Psychological Assessment Resources.

Blackshaw, A. J., Kinderman, P., Hare, D. J., \& Hatton, C. (2001). Theory of mind, causal attribution and paranoia in Asperger syndrome. Autism, 5, 147-163.

Blair, R. J., Frith, U., Smith, N., Abell, F., \& Cipolotti, L. (2002). Fractionation of visual memory: Agency detection and its impairment in autism. Neuropsychologia, 40, 108-118.

Bowler, D. M. (1992). "Theory of mind" in Asperger's syndrome. Journal of Child Psychology and Psychiatry, 33, 877-893.

Buss, D. M. (1992). Mate preference mechanisms: Consequences for partner choice and intrasexual competition. In J. H. Barkow, L. Cosmides, \& J. Tooby (Eds.), The adapted mind: Evolutionary psychology and the generation of culture (pp. 249-266). Oxford: Oxford University Press.

Castelli, F., Frith, C., Happé, F., \& Frith, U. (2002). Autism, Asperger syndrome and brain mechanisms for the attribution of mental states to animated shapes. Brain, 125, 1839-1849.

Frith, U. (2004). Emmanuel Miller lecture: Confusions and controversies about Asperger syndrome. Journal of Child Psychology and Psychiatry, $45,672-686$.

Gerland, G. (1997). A real person. Life from the outside. London: Souvenir Press, Translated from the Swedish.

Grelotti, D., Gauthier, I., \& Schultz, R. (2002). Social interest and the development of cortical face specialization: What autism teaches us about face processing. Developmental Psychobiology, 40, 213-225.

Happé, F. (1994). An advanced test of theory of mind ability: Understanding of story characters' thoughts and feelings by able autistics, mentally handicapped and normal children and adults. Journal of Autism and Developmental Disorders, 24, 129-154.

Happé, F., Ehlers, S., Fletcher, P., Frith, U., Johansson, M., Gillberg, C., Dolan, R., Frackowiak, R., \& Frith, C. (1996). 'Theory of mind' in the brain. Evidence from a PET scan study of Asperger syndrome. Neuroreport, 8, 197-201.

Heavey, L., Phillips, W., Baron-Cohen, S., \& Rutter, M. (2000). The Awkward Moments Test: A naturalistic measure of social understanding in autism. Journal of Autism and Developmental Disorders, 30, 225-236.

Hill, E., Berthoz, S., \& Frith, U. (2004). Brief report: Cognitive processing of own emotions in individuals with autistic spectrum disorder and their relatives. Journal of Autism and Developmental Disorders, 34, 229-235.

Hirschfeld, L. (1995). Do children have a theory of race? Cognition, 54, 209-252.

Hirschfeld, L. (1996). Race in the making: Cognition, culture, and the child's construction of human kinds. Cambridge: MIT Press.

Hobson, R. P. (1993). Autism and the development of mind. London: Erlbaum.

Joseph, R., \& Tanaka, J. (2003). Holistic and part-based face recognition in children with autism. Journal of Child Psychology and Psychiatry, 44, 529-542.

Kleinman, J., Marciano, P., \& Ault, R. (2001). Advanced theory of mind in high-functioning adults with autism. Journal of Autism and Developmental Disorders, 31, 29-36.

Klin, A., Jones, W., Schultz, R., Volkmar, F., \& Cohen, D. (2002). Visual fixation patterns during viewing of naturalistic social situations as predictors of social competence in individuals with autism. Archives of General Psychiatry, 59, 809-816.

Lawson, W. (1998). Life behind glass. A personal account of autism spectrum disorder. London: Jessica Kingsley Publishers Limited.

Macintosh, K., \& Dissanayake, C. (2004). Annotation: The similarities and differences between autistic disorder and Asperger's disorder: A review of the empirical evidence. Journal of Child Psychology and Psychiatry, 45, 421-434.

Mackie, D. M., Hamilton, D. L., Susskind, J., \& Rosselli, F. (1996). Social psychological foundations of stereotype formation. In C. N. Macrae, C. Stangor, \& M. Hewstone (Eds.), Stereotypes and stereotyping (pp. 41-78). New York: The Guilford Press.

O’Doherty, J., Winston, J., Critchley, H., Perrett, D., Burt, D., \& Dolan, R. (2003). Beauty in a smile: The role of medial orbitofrontal cortex in facial attractiveness. Neuropsychologia, 41, 147-155.

Perrett, D. I., May, K. A., \& Yoshikawa, S. (1994). Facial shape and judgements of female attractiveness. Nature, 368, 239-242.

Rutherford, M., Baron-Cohen, S., \& Wheelwright, S. (2002). Reading the mind in the voice: A study with normal adults and adults with Asperger syndrome and high functioning autism. Journal of Autism and Developmental Disorders, 32, 189-194.

Sainsbury, C. (2000). Martian in the playground: Understanding the school child with Asperger syndrome. Bristol: Lucky Duck Publishing. 
Shamay-Tsoory, S., Tomer, R., Yaniv, S., \& Aharon-Peretz, J. (2002). Empathy deficits in Asperger syndrome: A cognitive profile. Neurocase, 8, 245-252.

Silver, M., \& Oakes, P. (2001). Evaluation of a new computer intervention to teach people with autism or Asperger syndrome to recognize and predict emotions in others. Autism, 5, 299-316.

Wechsler, D. (1999). Wechsler Adult Intelligence Scale for Children (WAIS) (3rd UK ed.). London: Psychological Corporation.
Winston, J., Strange, B., O’Doherty, J., \& Dolan, R. (2002). Automatic and intentional brain responses during evaluation of trustworthiness of faces. Nature Neuroscience, 5, 277-283.

Ziatas, K., Durkin, K., \& Pratt, C. (1998). Belief term development in children with autism, Asperger syndrome, specific language impairment, and normal development: Links to theory of mind development. Journal of Child Psychology and Psychiatry, 39, 755-763. 